\title{
Infrared Laser Frequency Combs for Multispecies Gas Detection
}

\section{Vainio, Markku Mikael}

Springer

2017

Vainio , M M , Ulvila , V S \& Halonen , L O 2017 , Infrared Laser Frequency Combs for Multispecies Gas Detection . in M F Pereira \& O Shulika (eds) , THz for CBRN and

Explosives Detection and Diagnosis . NATO Science for Peace and Security Series B:

Physics and Biophysics, Springer, Dordrecht, pp. 151-158, NATO Advanced Research

pÿWorkshop on THz for CBRN and Explosives Detection and Diagnosis, Çe_me , Turkey , 03/11/2015 . https://doi.org/10.1007/978-94-024-1093-8_18

http://hdl.handle.net/10138/311036

https://doi.org/10.1007/978-94-024-1093-8_18

acceptedVersion

Downloaded from Helda, University of Helsinki institutional repository.

This is an electronic reprint of the original article.

This reprint may differ from the original in pagination and typographic detail.

Please cite the original version. 


\title{
Chapter 1 \\ Infrared Laser Frequency Combs for Multispecies Gas Detection
}

\author{
M. Vainio ${ }^{1,2}$, V. Ulvila ${ }^{1}$, L. Halonen ${ }^{1}$ \\ ${ }^{1}$ Laboratory of Physical Chemistry, Department of Chemistry, University of Helsinki, \\ Finland. markku.vainio@helsinki.fi.
}

${ }^{2}$ VTT Technical Research Centre of Finland Ltd, Centre for Metrology MIKES, Finland.

\begin{abstract}
We discuss two methods for mid-infrared frequency comb generation in quadratic nonlinear materials. The first one of these methods is based on halfharmonic generation in a degenerate femtosecond optical parametric oscillator, which is synchronously pumped with a near-infrared laser frequency comb. The second method uses a continuous-wave-pumped optical parametric oscillator. In this approach, the quadratic nonlinearity is used not only for frequency down conversion, but also for the actual frequency comb generation from a continuouswave pump laser field. Both methods can be used to produce coherent broadband mid-infrared light that is ideal for real-time spectroscopic detection of small traces of biomarkers, toxics, and other interesting molecular compounds. In particular, these methods provide access to the 3 to $5 \mu \mathrm{m}$ molecular fingerprint region, which contains strong fundamental vibrational stretching bands of hydrocarbons.
\end{abstract}

Keywords: Optical frequency comb, Laser frequency comb, Nonlinear optics, Optical parametric oscillator, Infrared spectroscopy

\subsection{Introduction}

An optical frequency comb (OFC) generator produces coherent broadband laser light that has a spectrum consisting of narrow equidistant lines. For an OFC suitable for high-resolution molecular spectroscopy, the comb tooth spacing is typically of the order of $100 \mathrm{MHz}$, and the number of comb teeth can be up to several hundred thousand. In the visible and near-infrared regions octave spanning fre- 
quency combs are routinely produced by mode-locked femtosecond lasers followed by additional spectral broadening in a highly nonlinear optical fiber. These combs can be fully stabilized by locking the comb offset frequency and repetition rate (tooth spacing) to a stable microwave frequency reference. The invention of fully stabilized OFCs in 1999 has led to several new high-accuracy laser spectroscopy methods [1]. As an example, dual-comb spectroscopy provides outstanding speed, accuracy, and spectral resolution in simultaneous detection of multiple molecular species [2,3]. The discrete broadband nature of OFC light also allows for spectroscopy of molecules that have wide and complex spectra, and are therefore difficult to reliably detect with conventional lasers. Optical frequency combs can be combined with state-of-the-art laser spectroscopy techniques, such as cavityenhanced spectroscopy, which allows parts-per-billion (ppb) level detection limits in real-time molecular spectroscopy $[4,5]$.

The mid-infrared region is in many cases the optimal spectral region for molecular spectroscopy, but OFCs in this region are scarce. Direct generation of OFCs at wavelengths longer than $2.8 \mu \mathrm{m}$ by mode-locked lasers is yet to be demonstrated. Quantum cascade laser frequency combs have been investigated in the long mid-infrared (approximately 7 to $10 \mu \mathrm{m}$ ) and terahertz regions, while the 3 to $5 \mu \mathrm{m}$ spectral window can be accessed by methods based on nonlinear optics $[6,7]$. The basic idea of these methods is to use nonlinear frequency conversion to transfer a near-infrared OFC to the mid-infrared region. One of the best approaches for this is synchronously pumped degenerate femtosecond optical parametric oscillator (OPO), which is also referred to as half-harmonic generator [8-10]. The degenerate femtosecond OPO produces a mid-infrared frequency comb that is inherently phase-locked to the pump comb. The instantaneous spectral bandwidth of a degenerate fs-OPO can be hundreds of nanometers, and the average output power tens of milliwatts. A more detailed description and the results of recent research of our group on this method are given in Section 1.3.

In Section 1.4, we focus on so-called CACHI comb generation, which is a new nonlinear optics approach for frequency comb generation [11-13]. While the other quadratic nonlinear optics methods work merely as frequency down-converters that require a femtosecond OFC as an input, the CASCHI method uses cascaded quadratic nonlinearities to actually generate the frequency comb from a continuous-wave pump field. This new solution is suitable for direct mid-infrared frequency comb generation, but it can also be combined with nonlinear downconversion, in which case a simple near-infrared CW pump laser can be used to produce a comb in the 3-5 $\mu \mathrm{m}$ region [14]. Here, we demonstrate generation of a composite CASCHI comb with a single instrument from blue to mid-infrared. The mid-infrared output power is up to several watts, which exceeds the power levels of most other infrared OFCs by one to three orders of magnitude. 


\subsection{Optical Parametric Oscillator (OPO)}

An optical parametric oscillator (OPO) is a frequency down-converter, which generates two output frequencies, signal $\left(v_{\mathrm{s}}\right)$ and idler $\left(v_{\mathrm{i}}\right)$, in a nonlinear crystal when pumped with an intense laser beam at frequency $v_{p}$. The crystal is placed inside an optical resonator, which is configured either as singly resonant $\left(v_{\mathrm{s}}\right.$ or $v_{\mathrm{i}}$ resonating), or doubly resonant (both $v_{\mathrm{s}}$ and $v_{\mathrm{i}}$ resonating). The OPO process needs to conserve both photon energy and momentum, which respectively leads to two equations that both need to be fulfilled:

$v_{i}=v_{p}-v_{s}$

$\Delta k=k_{p}-k_{s}-k_{i}=0$,

where $k_{\mathrm{x}}=2 \pi n_{\mathrm{x}} / \lambda_{\mathrm{x}}$ is the angular wavenumber, with $n_{\mathrm{x}}$ being the refractive index of the crystal material at the respective wavelength $\lambda_{\mathrm{x}}$.

Equation 2 is often referred to as phase matching condition. Phase matching for generation of mid-infrared light at wavelength $\lambda_{\mathrm{i}}=c / \nu_{\mathrm{i}} \sim 3 \mu \mathrm{m}$ with nearinfrared pumping is typically obtained by quasi-phase matching, for example by using a periodically poled lithium niobate crystal (PPLN).

\subsection{Synchronously-pumped Degenerate Femtosecond OPO}

When an OPO is pumped with a mode-locked femtosecond laser whose output spectrum is a frequency comb, also the generated signal and idler spectra are combs [8]. A special case is a degenerate fs-OPO, where the signal and idler spectra overlap, such that they become indistinguishable via mutual injection locking (Figure 1). The degenerate fs-OPO thus works as a half-harmonic generator. It has been shown that the output of a degenerate fs-OPO is inherently phase-locked to the pump comb, with two alternative, discrete states [8].

Figure 1 shows schematically the experimental setup of the degenerate fs-OPO developed in our laboratory. The OPO is pumped with $450 \mathrm{~mW}$ average power from a fully-stabilized Er-fiber laser comb (Menlo Systems $\mathrm{GmbH}$ ), which has a $-3 \mathrm{~dB}$ bandwidth of about $50 \mathrm{~nm}$ centered at $1560 \mathrm{~nm}$. The repetition frequency of pump pulses is $250 \mathrm{MHz}$, and the pulse length $<300 \mathrm{fs}$. The pump pulses are tightly focused in a 1-mm long Brewster-cut MgO-doped PPLN crystal, which is placed inside the OPO cavity. The cavity mirrors are gold-coated, except for the input coupler, which is a dielectric mirror that transmits the pump beam but is highly reflective for the signal/idler mid-infrared wavelengths. A thin pellicle beam splitter is used as a partial $(\sim 10 \%)$ output coupler for the mid-infrared beam. 

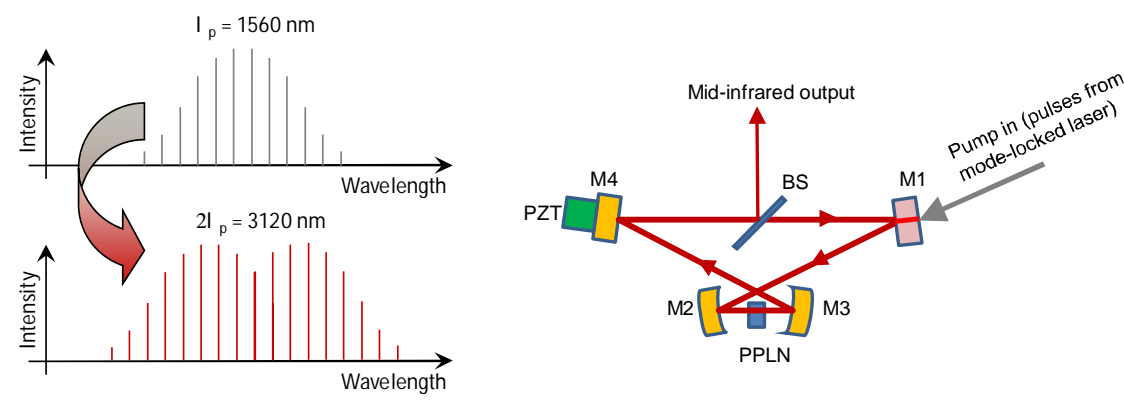

Figure 1 Left: The principle of comb generation by degenerate fs-OPO that divides the center wavelength of the pump comb by two. In reality, the number of comb teeth is tens of thousands. Right: A schematic illustration of the fs-OPO cavity. Mirror M1 is the pump input coupler, and mirrors M2-M4 are gold-coated mirrors. Up to $20 \mathrm{~mW}$ of the resonating mid-infrared beam is coupled out with a beam splitter (BS). A piezo-electric actuator (PZT) is used for cavity length fine tuning and stabilization.

Owing to the short duration of the pump pulses, the free spectral range of the OPO cavity needs to be synchronized with the pump repetition frequency, such that the mid-infrared pulses resonating in the cavity overlap with consecutive pump pulses at each round trip. This condition sets the cavity length of our OPO to $c / 250 \mathrm{MHz}=1.199 \mathrm{~m}$. A piezoelectric actuator attached to one of the cavity mirrors is used to fine-tune the cavity length with a resolution better than $1 \mu \mathrm{m}$, which is needed to match the resonance condition of the synchronously pumped degenerate OPO. We use active locking to stabilize the OPO cavity length for long-term operation of several hours. The average mid-infrared output power of the stabilized fs-OPO is between 3 and $20 \mathrm{~mW}$, depending on the pump power and output coupler used in the experiments.

The output spectrum of the degenerate fs-OPO can be modified by fine-tuning the OPO cavity length $[9,10]$. The fully degenerate state, in which also the comb offset frequency is tightly locked to the pump comb, is the most favorable for applications like dual-comb spectroscopy, which requires high phase coherence and stability. Two fs-OPO output spectra corresponding to such degeneracy are exemplified in Figure 2. Absorption features of compounds in the laboratory air are seen, not just due to the absorption between the OPO and the spectrum analyzer, but also owing to the absorption inside the OPO cavity. In fact, the OPO cavity increases the effective absorption path length by a factor of 5 to 10, leading to a respective enhancement in gas detection sensitivity [15]. 


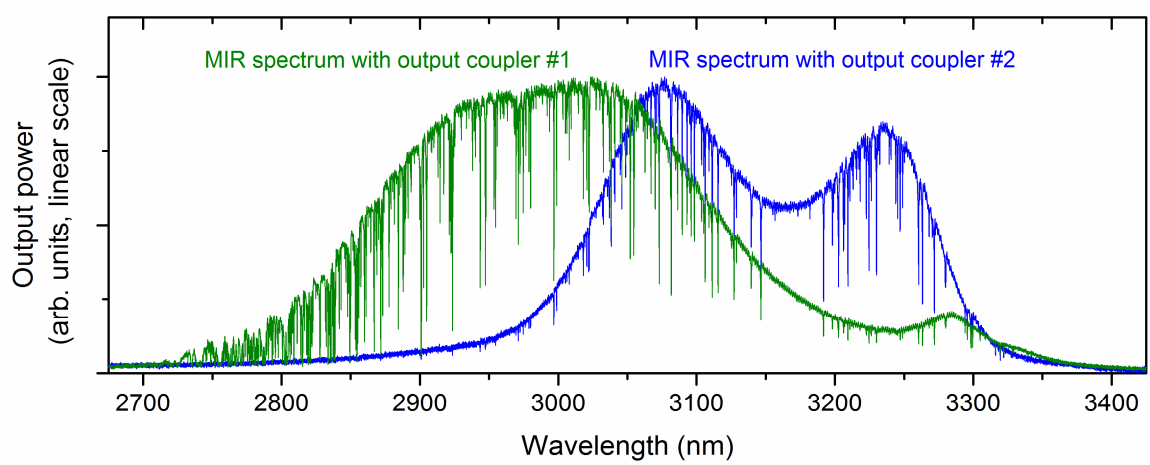

Figure 2 Output spectrum of the fs-OPO measured with two different output couplers. Note that the comb tooth spacing ( $250 \mathrm{MHz}$, or $\sim 8 \mathrm{pm}$ ) is too small to be resolved in the figure. The narrow peaks pointing downwards in the spectra are due to absorption of light in laboratory air over an effective absorption path length of $13 \mathrm{~m}$. The absorption is mostly due to water vapor, but peaks resulting from $\sim 2$ ppm of $\mathrm{CH}_{4}$ in air are also observed with a signal-to-noise ratio of $>10$ (limited by etalon effects). Both spectra were measured with a Fourier-transform infrared spectrometer (Bruker IFS $120 \mathrm{HR}$ ).

\subsection{Continuous-Wave-Pumped CASCHI Comb Generation}

The principle of optical frequency comb generation by cascaded quadratic nonlinearities is illustrated in Figure 3 and explained in more detail in references [1114]. In brief, the comb generation process from a CW pump field includes two steps: cavity-enhanced second-harmonic generation, and subsequent back conversion, where the back-converted light fills the pump resonator cavity modes while obeying the laws of energy and momentum conversion (Eqs. 1 and 2). So far the most accurate measurements of the CASCHI comb line spacing have confirmed the comb equidistance at $1 \mathrm{~Hz}$ level [13].

We have implemented the CASCHI scheme inside a CW-pumped OPO, which inherently transfers the frequency comb from near-infrared to mid-infrared [14]. In addition, residual nonlinear mixing processes extend the comb to higher frequencies, all the way to the blue, as demonstrated in Figure 4. The composite frequency comb produced by the combination of various nonlinear processes covers three octaves, from $400 \mathrm{~nm}$ to $3.5 \mu \mathrm{m}$. 


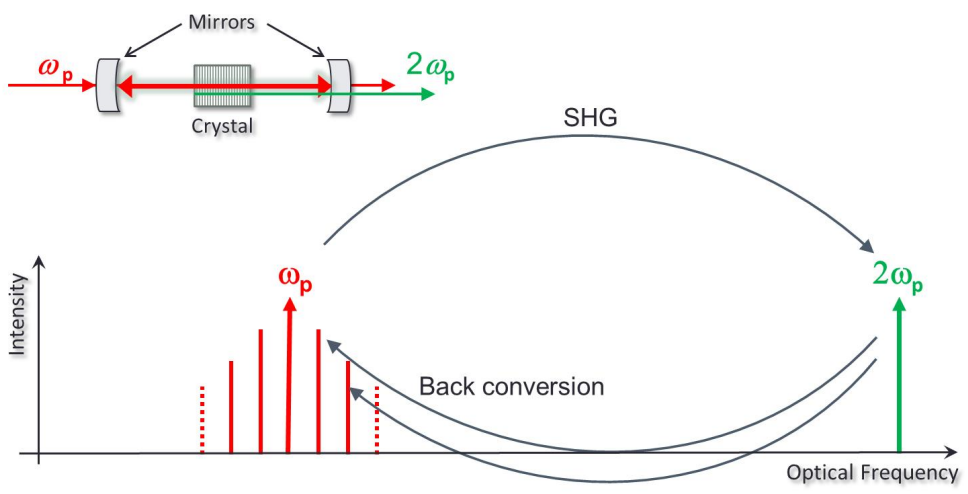

Figure 3 The principle of CASCHI comb generation. A nonlinear crystal is placed inside an optical resonator. The crystal is designed for second harmonic generation (SHG) of the pump beam. With a properly adjusted phase-mismatch efficient back-conversion (cascading nonlinear effect) of the second harmonic light takes place, generating new frequency comb components close to the original pump frequency. The frequency separation of the comb teeth is determined by the length of the resonator.

One of the advantages of the CASCHI comb is the possibility of achieving high, multiwatt output powers in the mid-infrared region. We have demonstrated up to $3 \mathrm{~W}$ of output power in the $3 \mu \mathrm{m}$ fingerprint region [14], and more than $4 \mathrm{~W}$ in the $2 \mu \mathrm{m}$ region [13] using CW near-infrared laser pumping. While the instantaneous bandwidth of the first CASCHI prototype mid-infrared comb is limited to approximately $10 \mathrm{~nm}$, the center wavelength of the comb can easily be tuned over a wide range in the molecular fingerprint region, as shown in Figure 5.

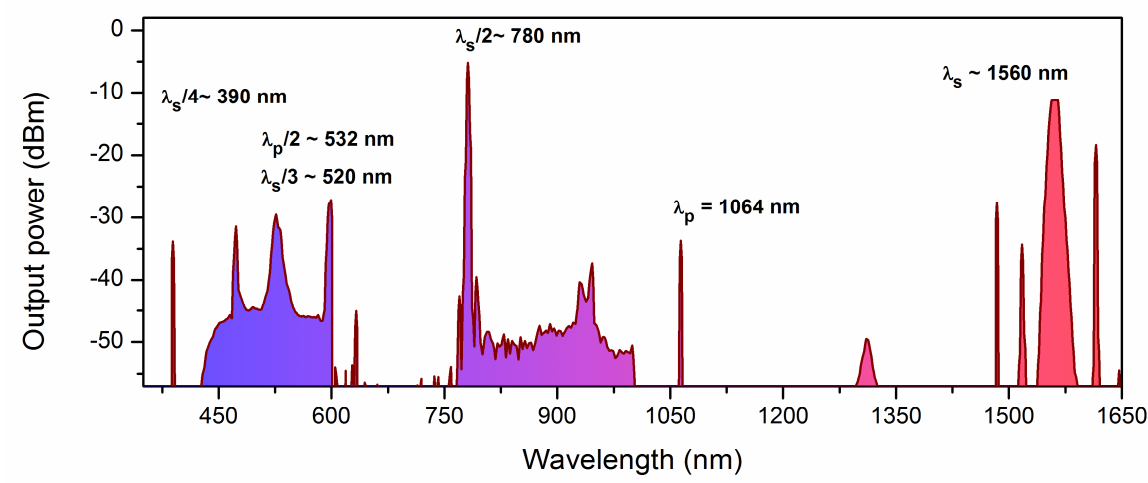

Figure 4 Envelope spectrum of a composite CASCHI frequency comb generated by cascaded quadratic nonlinearities inside a CW OPO, which is pumped at $1064 \mathrm{~nm}$ [14]. Indexes p and s refer to OPO pump and signal wavelengths, respectively. The mid-infrared comb around the idler wavelength at $>3 \mu \mathrm{m}$ is outside the measurement range of the spectrum analyzer used here. Examples of the midinfrared comb spectra, as measured with another spectrum analyzer, are shown in Fig. 5. 


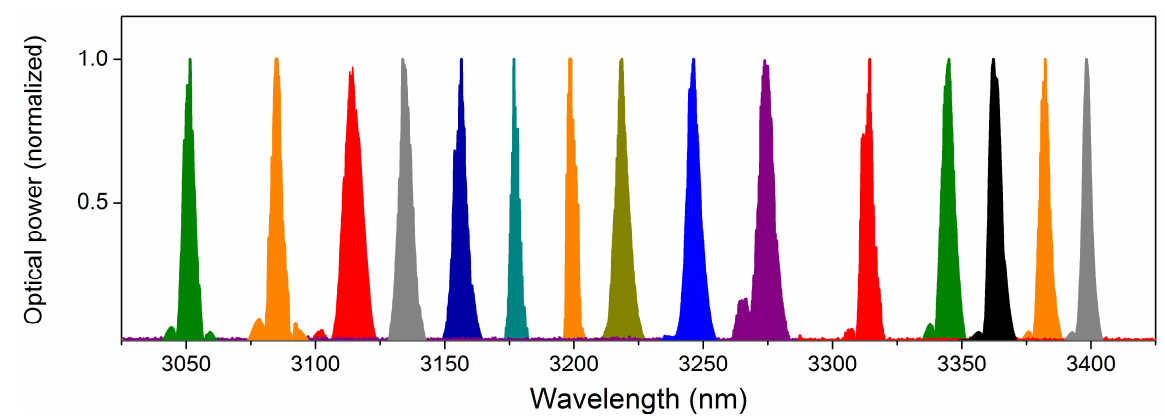

Figure 5 Mid-infrared CASCHI comb envelope spectra measured with different phase-matching conditions of the $\mathrm{CW}$ OPO. Tuning of the comb center wavelength was done by changing the temperature and poling period of the OPO crystal [14]. The comb tooth spacing is not resolved in the figure.

\section{Conclusion}

In conclusion, we have reported two experimental approaches based on quadratic optical nonlinearities to generate optical frequency combs in the 3 to $5 \mu \mathrm{m}$ molecular fingerprint region. The first approach relies on a degenerate, synchronously pumped femtosecond OPO, which phase-coherently transfers a near-infrared frequency comb to the mid-infrared region. The instantaneous spectral coverage of the comb is hundreds of nanometers.

Our second approach for mid-infrared frequency comb generation is based on cascaded quadratic nonlinearities. This CASCHI method allows for comb generation practically at any wavelength from visible to mid-infrared with simple $\mathrm{CW}$ laser pumping. The output power can be up to several watts. In future, miniaturization of the CASCHI comb for field applications will be investigated [16].

Acknowledgments: We thank the Academy of Finland, the Finnish Funding Agency for Innovation (Tekes), and the University of Helsinki for financial support.

\section{References}

1. S. A. Diddams, The evolving optical frequency comb. JOSA B 27, B51-B62 (2010). doi: 10.1364/JOSAB.27.000B51,

2. S. Schiller, Spectrometry with frequency combs. Opt. Lett. 27, 766-768 (2002). doi: 10.1364/OL.27.000766,

3. I. Coddington, W. Swann, N. Newbury, Coherent multiheterodyne spectroscopy using stabilized optical frequency combs. Phys. Rev. Lett. 100, 013902 (2008). doi: 10.1103/PhysRevLett.100.013902, 
4. F. Adler, M. J. Thorpe, K. C. Cossel, J. Ye, Cavity-Enhanced Direct Frequency Comb Spectroscopy: Technology and Applications. Annu. Rev. Anal. Chem. 3, 175-205 (2010). doi: 10.1146/annurev-anchem-060908-155248,

5. A. Khodabakhsh, V. Ramaiah-Badarla, L. Rutkowski, A. C. Johansson, K. F. Lee, J. Jiang, C. Mohr, M. E. Fermann, A. Foltynowicz, Optical frequency comb spectroscopy at 3-5.4 $\mu \mathrm{m}$ with a doubly resonant optical parametric oscillator. arXiv:1603.09680 (2016).

6. A. Schliesser, N. Picqué, T. W. Hänsch, Mid-infrared frequency combs. Nat. Photonics 6, 440-449 (2012). doi:10.1038/nphoton.2012.142,

7. M. Vainio, L. Halonen, Mid-infrared optical parametric oscillators and frequency combs for molecular spectroscopy. Phys. Chem. Chem. Phys. 18, 4266-4294(2016). doi: 10.1039/c5cp07052j,

8. S. T. Wong, K. L. Vodopyanov, R. L. Byer, Self-phase-locked divide-by-2 optical parametric oscillator as a broadband frequency comb source. J. Opt. Soc. Am. B 27, 876-882 (2010). doi: 10.1364/JOSAB.27.000876,

9. N. Leindecker, A. Marandi, R. L. Byer, K. L. Vodopyanov, Broadband degenerate OPO for mid-infrared frequency comb generation. Opt. Express 19, 62966302 (2011). doi: 10.1364/OE.19.006296,

10. M. Vainio, M. Merimaa, L. Halonen, K. L. Vodopyanov, Degenerate $1 \mathrm{GHz}$ repetition rate femtosecond optical parametric oscillator. Opt. Lett. 37, 45614563 (2012). doi: 10.1364/OL.37.004561,

11. V. Ulvila, C. R. Phillips, L. Halonen, M. Vainio, Frequency comb generation by a continuous-wave-pumped optical parametric oscillator based on cascading quadratic nonlinearities. Opt. Lett. 38, 4281-4284 (2013). doi: 10.1364/OL.38.004281,

12. I. Ricciardi, S. Mosca, M. Parisi, P. Maddaloni, L. Santamaria, P. De Natale, M. DeRosa, Frequency comb generation in quadratic nonlinear media. Phys. Rev. A 91, 063839 (2015). doi: 10.1103/PhysRevA.91.063839

13. V. Ulvila, C. R. Phillips, L. Halonen, M. Vainio, Spectral characterization of a frequency comb based on cascaded quadratic nonlinearities inside an optical parametric oscillator. Phys. Rev. A 92, 033816 (2015). doi: 10.1103/PhysRevA.92.033816,

14. V. Ulvila, C. R. Phillips, L. Halonen, M. Vainio, High-power mid-infrared frequency comb from a continuous-wave-pumped bulk optical parametric oscillator. Opt. Express 22, 10535-10543 (2014). doi: 10.1364/OE.22.010535,

15. M. W. Haakestad, T. P. Lamour, N. Leindecker, A. Marandi, K. L. Vodopyanov, Intracavity trace molecular detection with a broadband mid-IR frequency comb source. J. Opt. Soc. Am. B 30, 631-640 (2013). doi: 10.1364/JOSAB.30.000631,

16. F. Leo, T. Hansson, I. Ricciardi, M. De Rosa, S. Coen, S. Wabnitz, M. Erkintalo, Walk-off-induced modulation instability, temporal pattern formation, and frequency comb generation in cavity-enhanced second-harmonic generation. arXiv:1510.04261 (2015). 